\title{
STRAIN DIFFERENCES IN BAROCEPTOR REFLEX IN ADULT WISTAR KYOTO RATS
}

\author{
Vitor E. Valenti, ${ }^{\text {L Luiz Carlos de Abreu, }{ }^{\text {II }} \text { Caio Imaizumi, }}$ Márcio Petenusso, ${ }^{\text {II }}$ \\ Celso Ferreira, ${ }^{\mathrm{I}}$ III
}

doi: $10.1590 / \mathrm{S} 1807-59322010000200013$

\begin{abstract}
Valenti VE, de Abreu LC, Imaizumi C, Ferreira C. Differences in baroreceptor reflexes in adult wistar kyoto rats. Clinics. 2010;65(2):203-8.
\end{abstract}

OBJECTIVES: A subset of normotensive Sprague-Dawley rats show lower baroreflex sensitivity; however, no previous study investigated whether there are differences in baroreflex sensitivity within this subset. Our study compared baroreflex sensitivity among conscious rats of this specific subtype.

METHODS: Male Wistar Kyoto (WKY) rats (16 weeks old) were studied. Cannulas were inserted into the abdominal aortic artery through the right femoral artery to measure mean arterial pressure (MAP) and heart rate (HR). Baroreflex gain was calculated as the ratio between change in $\mathrm{HR}$ and MAP variation $(\triangle \mathrm{HR} / \triangle \mathrm{MAP})$ in response to a depressor dose of sodium nitroprusside (SNP, $50 \mu \mathrm{g} / \mathrm{kg}$, i.v.) and a pressor dose of phenylephrine (PE, $8 \mu \mathrm{g} / \mathrm{kg}$, i.v.). Rats were divided into four groups: 1) low bradycardic baroreflex (LB), baroreflex gain (BG) between -1 and $-2 \mathrm{bpm} / \mathrm{mmHg}$ tested with PE; 2) high bradycardic baroreflex (HB), BG $<-2$ $\mathrm{bpm} / \mathrm{mmHg}$ tested with PE; 3) low tachycardic baroreflex (LT), BG between -1 and $-2 \mathrm{bpm} / \mathrm{mmHg}$ tested with SNP and; 4) high tachycardic baroreflex (HT), BG $<-2 \mathrm{bpm} / \mathrm{mmHg}$ tested with SNP. Significant differences were considered for $\mathrm{p}<0.05$.

RESULTS: Approximately $37 \%$ of the rats showed a reduced bradycardic peak, bradycardic reflex and decreased bradycardic gain of baroreflex while roughly $23 \%$ had a decreased basal HR, tachycardic peak, tachycardic reflex and reduced sympathetic baroreflex gain. No significant alterations were noted with regard to basal MAP.

CONCLUSION: There is variability regarding baroreflex sensitivity among WKY rats from the same laboratory.

KEYWORDS: Baroreflex; Rats; Inbred WKY; Sympathetic Nervous System; Parasympathetic Nervous System; Autonomic Nervous System.

\section{INTRODUCTION}

Okamoto and Aoki ${ }^{1}$ in 1963 selectively bred Wistar rats to spontaneously develop hypertension. Recognized as an inbred strain in 1969 by the National Institutes of Health (NIH), the spontaneously hypertensive rat (SHR) is accepted as the most studied animal model of essential hypertension. As controls for experiments utilizing SHRs, many scientists have employed normotensive descendants of Wistar rats that

\footnotetext{
${ }^{\mathrm{I}}$ Department of Medicine, Cardiology Division, Federal Unversity of São Paulo (UNIFESP) - São Paulo/SP, Brazil.

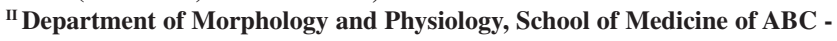
Santo André/SP, Brazil.

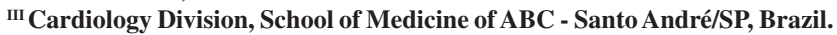
Email: valenti@unifesp.br

Tel.: 55115572.5462

Received for publication on October 09, 2009

Accepted for publication on November17, 2009
}

NIH investigators obtained in 1971 from a colony in Kyoto from which the SHR strain was originally derived (WistarKyoto rats, WKY). ${ }^{2}$

The baroreflex is one of the body's homeostatic mechanisms for sustaining blood pressure. Modifications in baroreflex control of the heart have been shown to be related to a predisposition for increased cardiac mortality and sudden cardiac death. As a result, the baroreflex has garnered considerable interest. The evaluation of baroreflex sensitivity is an established tool for the assessment of autonomic control of the cardiovascular system. Aside from the wellacknowledged physiological role in the maintenance of circulatory homeostasis, accumulating evidence suggests that changes in the characteristics of baroreflex function reflect alterations in the autonomic control of the cardiovascular system. Thus, the baroreflex has been shown to be a source of valuable information in the clinical management, 
particularly in prognostic evaluation and assessment of treatment effect, of a variety of cardiac diseases. ${ }^{3-6}$

Although it was previously reported that some normotensive Sprague-Dawley rats spontaneously exhibit lower baroreflex sensitivity, ${ }^{7,10}$ no previous study investigated whether there is difference in baroreflex sensitivity among other types of rats from the same parent strain. In addition, a previous study demonstrated that WKY rats from two different laboratories showed differences in arterial pressure and growth rate. ${ }^{11}$ Thus, in this study we aimed to compare baroreflex function among adult WKY rats from the same laboratory in order to identify any intra-strain differences.

\section{METHODS}

\section{Animals}

The experiments were performed on 16-week-old male WKY rats from the same initial laboratory. Rats were housed individually in plastic cages under standard laboratory conditions. They were kept under a $12 \mathrm{~h}$ light/dark cycle (lights on at 6:30 am) and had free access to food and water. Housing conditions and experimental procedures were approved by the institution's Animal Ethics Committee. Efforts were made to minimize the number of animals used.

\section{Surgical Preparation}

One day before the experiments, the rats were anesthetized with ketamine $(50 \mathrm{mg} / \mathrm{kg}$ i.p. $)$ and xylaxine $(50$ $\mathrm{mg} / \mathrm{kg}$ i.m.) and a catheter was inserted into the abdominal aorta through the femoral artery for blood pressure and heart rate recording. Catheters were made of $4 \mathrm{~cm}$ segments of PE-10 polyethylene (Clay Adams, USA) heat-bound to a 13 $\mathrm{cm}$ segment of PE-50. The catheters were tunneled under the skin and exteriorized at the animal's dorsum. ${ }^{12}$

\section{Arterial pressure and heart rate recording in conscious rats}

Approximately 24 hours after surgery, the animals were placed in individual cages used for transport to the experimental room. Animals were allowed $60 \mathrm{~min}$ to adapt to the conditions of the experimental room before starting blood pressure and heart rate recording. The experimental room was acoustically isolated and had constant background noise produced by an air exhauster. A minimum of 30 additional minutes was allowed before beginning experiments. Pulsatile arterial pressure (PAP) of freely moving animals was recorded using an HP-7754A preamplifier (Hewlett Packard, USA) and an acquisition board (MP100A, Biopac Systems Inc, USA) connected to a computer. Mean arterial pressure (MAP) and heart rate (HR) values were derived from the PAP recordings and processed online. ${ }^{12}$ No signs of pain or discomfort were observed.

\section{Baroreflex test}

The baroreflex was tested with a pressor dose of phenylephrine (PE, bolus $8 \mu \mathrm{g} / \mathrm{kg}$ i.v.; Sigma Chemical) and vasodilator dose of sodium nitroprusside (SNP, bolus $50 \mu \mathrm{g} / \mathrm{kg}$ i.v.; RBI). Baroreflex gain was calculated as the ratio between the change in HR and MAP variation ( $\triangle \mathrm{HR} / \Delta \mathrm{MAP})$. There was an interval of at least 15 minutes between infusions to allow the recovery of basal values. We also evaluated bradycardic and tachycardic peaks and HR range, i.e., the difference between bradycardic and tachycardic peaks. ${ }^{12}$ We separated rats into groups according to baroreflex gain (BG): 1) low bradycardic baroreflex (LB), BG between -1 and $-2 \mathrm{bpm} / \mathrm{mmHg}$ tested with PE; 2) high bradycardic baroreflex (HB), BG $<-2 \mathrm{bpm} / \mathrm{mmHg}$ tested with PE; 3) low tachycardic baroreflex (LT), BG between -1 and $-2 \mathrm{bpm} / \mathrm{mmHg}$ tested with SNP and; 4) high tachycardic baroreflex (HT), BG $<-2 \mathrm{bpm} / \mathrm{mmHg}$ tested with SNP. We compared the LB group with the HB group and the LT group with the HT group. We defined the values for bradycardic and tachycardic baroreflex gain according to pilot studies.

\section{Statistical analysis}

Values were reported as the means \pm standard error of means (S.E.M.). HR, MAP, $\Delta \mathrm{HR}, \triangle \mathrm{MAP}$ and $\Delta \mathrm{HR} / \Delta \mathrm{MAP}$ were compared between LB and HB groups as well as between LT and HT groups. After the distributions were evaluated using the Kolmogorov normality test, Student's $T$ test was used to verify differences between data with normal distributions and the Mann-Whitney test was applied to assess differences between non-parametric distributions. Differences were considered significant when the probability of a Type I error was less than 5\% ( $\mathrm{p}<0.05)$.

\section{RESULTS}

Among the 22 WKY rats evaluated based on bradycardic baroreflex gain, approximately $63 \%$ had high bradycardic baroreflex gain (HB; $<-2 \mathrm{bpm} / \mathrm{mmHg}$ ). On the other hand, a small portion of the animals exhibited lower bradycardic baroreflex gain (LB; between -1 and $-2 \mathrm{bpm} / \mathrm{mmHg}$ ).

We also compared baseline MAP and HR, bradycardic and tachycardic peaks, HR range and parasympathetic and sympathetic baroreflex gain. As seen in Table 1, significant differences in bradycardic peak and the 
parasympathetic component of baroreflex gain, but not in the other parameters, were observed.

Table 1- Baseline mean arterial pressure (MAP) and heart rate (HR), bradycardic and tachycardic peaks, HR range, parasympathetic (PBG) and sympathetic baroreflex gain (SBG) in HB $(n=14)$ and LB $(n=8)$ groups. Mean \pm SEM.

\begin{tabular}{lccc}
\hline Variable & LB & HB & p value \\
\hline MAP (mmHg) & $119.12 \pm 5.3$ & $113.6 \pm 1.7$ & 0.2341 \\
HR (bpm) & $330.12 \pm 7.7$ & $342.8 \pm 13$ & 0.4 \\
Bradycardic peak (bpm) & $236.7 \pm 7.9$ & $191.8 \pm 6.9$ & 0.005 \\
Tachycardic peak (bpm) & $458.25 \pm 10.1$ & $455.8 \pm 13.2$ & 0.9 \\
HR range (bpm) & $221.5 \pm 5.3$ & $264 \pm 17.5$ & 0.08 \\
PBG (bpm x mmHg (bHE $^{-1}$ PH & $-1.41 \pm 0.12$ & $-2.63 \pm 0.11$ & $<0.0001$ \\
SBG (bpm x mmHg SNP $^{-1}$ & $-2.96 \pm 0.14$ & $-2.7 \pm 0.23$ & 0.458 \\
\hline
\end{tabular}

The PE-induced increase in MAP was not different between LB and HB groups ( $\mathrm{p}=0.75$ ). On the other hand, bradycardic reflex responses to intravenous $\mathrm{PE}$ were significantly decreased in the LB group $(\mathrm{p}=0.0006)$ (Figure 1).

We also compared the SNP-induced decrease in MAP
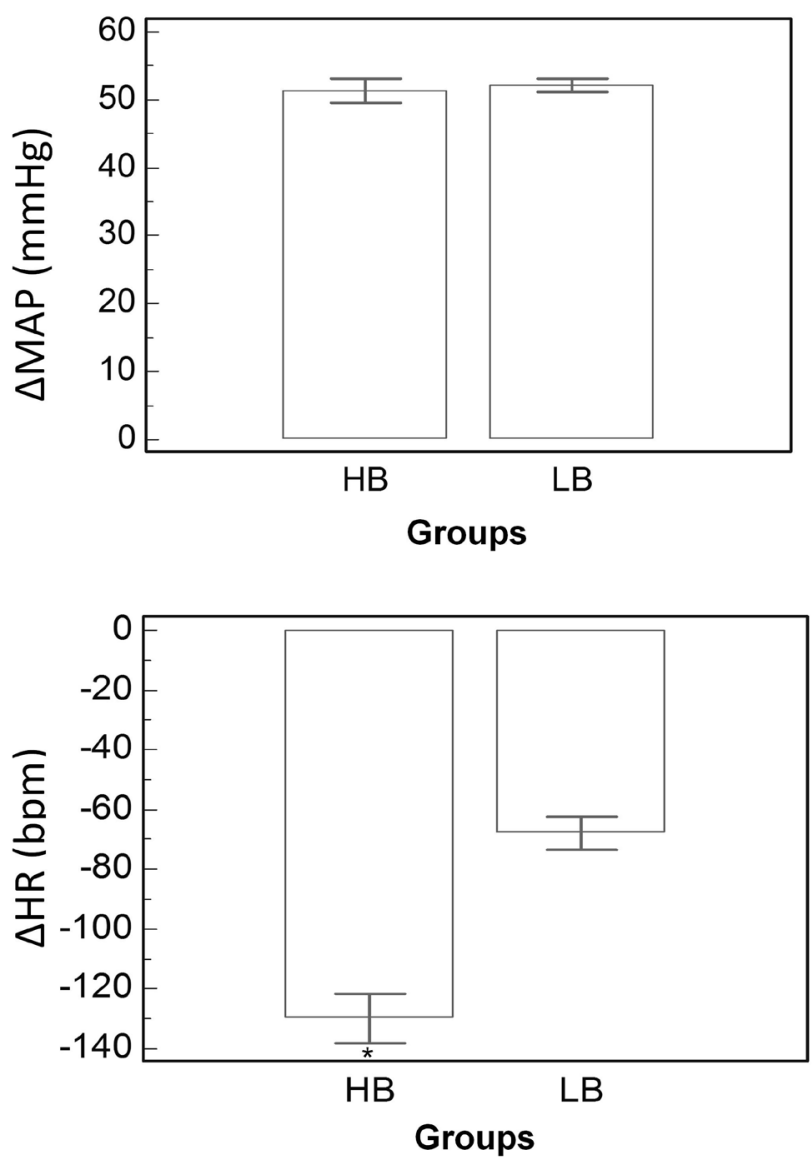

Figure 1 - Increase in mean arterial pressure (MAP, $\mathrm{mmHg}$ ) and decrease in heart rate $(\mathrm{HR}, \mathrm{bpm})$ in response to phenylephrine $(\mathrm{PE}, 8 \mu \mathrm{g} / \mathrm{kg}$ i.v.) in $\mathrm{HB}(\mathrm{n}=14)$ and LB $(\mathrm{n}=8)$ groups. ${ }^{\mathrm{p}}<0.001$ : difference in LB. Means \pm SEM.
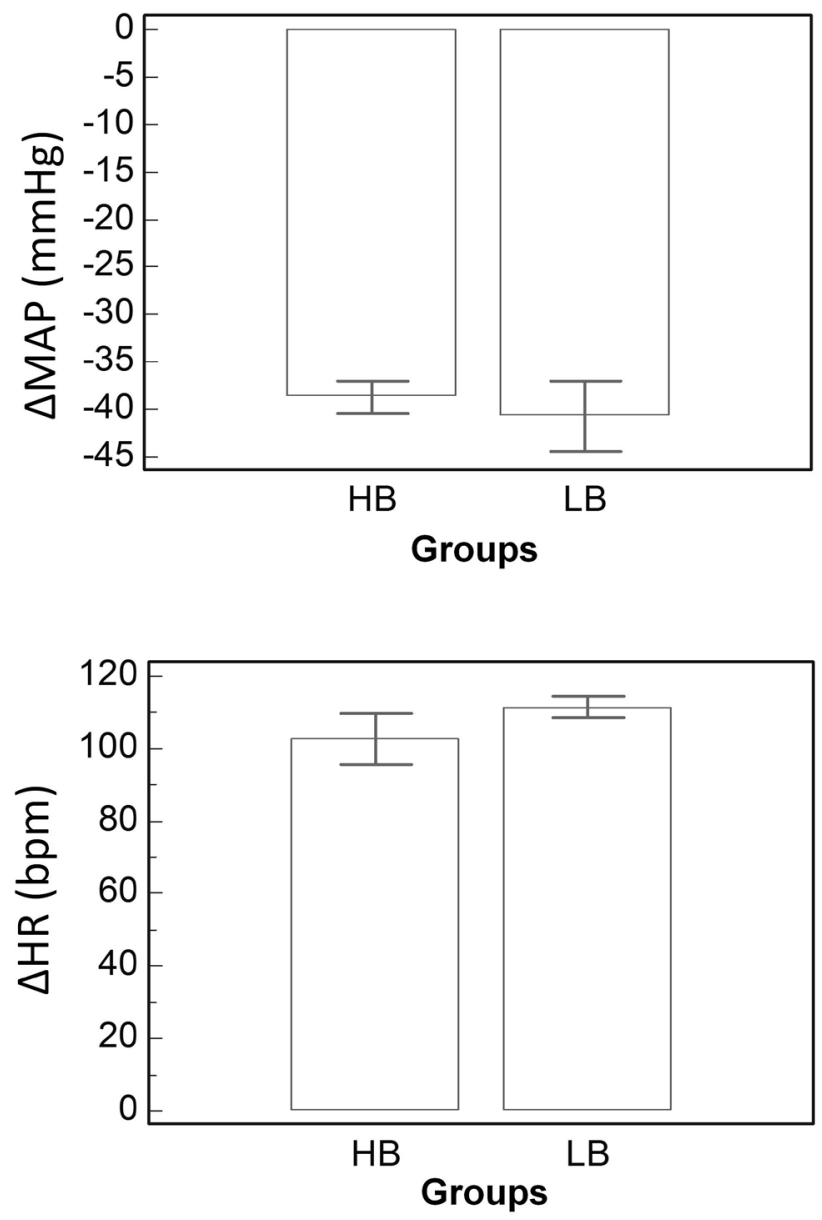

Figure 2 - Decrease in mean arterial pressure (MAP, $\mathrm{mmHg}$ ) and decrease in heart rate (HR, bpm) in response to sodium nitroprusside (SNP, $50 \mu \mathrm{g} / \mathrm{kg}$ i.v. $)$ in $\mathrm{HB}(\mathrm{n}=14)$ and LB $(\mathrm{n}=8)$ groups. Means \pm SEM.

and tachycardic reflex responses between $\mathrm{LB}$ and $\mathrm{HB}$ groups. The MAP decrease in the response to SNP was similar between the groups $(\mathrm{p}=0.58)$, as were the tachycardic reflex responses ( $\mathrm{p}=0.38$ ) (Figure 2).

When baroreflex gain was tested with SNP, we noted that among the $26 \mathrm{WKY}$ rats analyzed, approximately $77 \%$ showed higher baroreflex gain (HT; <-2 bpm/mmHg) while the remainder (around 23\%) were found to have lower baroreflex gain (LT; between -1 and $-2 \mathrm{bpm} / \mathrm{mmHg}$ ).

Baseline MAP and HR, bradycardic and tachycardic peak, HR range and parasympathetic and sympathetic baroreflex gain were compared between HT and LT groups. As shown in Table 2, we observed significant differences with respect to basal HR, tachycardic peak, HR range and sympathetic baroreflex gain.

The PE-induced increase in MAP was significantly higher in the HT group $(\mathrm{p}<0.001)$.

However, bradycardic reflex responses to an increase in arterial pressure were not different between the groups $(\mathrm{p}=0.143)$ (Figure 3). 
Table 2- Baseline mean arterial pressure (MAP) and heart rate (HR), bradycardic and tachycardic peak, HR range, parasympathetic (PBG) and sympathetic baroreflex gain (SBG) in HT $(n=20)$ and LT $(n=6)$ groups. Mean \pm SEM.

\begin{tabular}{|c|c|c|c|}
\hline Variable & LT & HT & p value \\
\hline MAP (mmHg) & $118.3 \pm 1.1$ & $115.05 \pm 2.4$ & 0.47 \\
\hline HR (bpm) & $236 \pm 1.59$ & $348.1 \pm 5.9$ & $<0.0001$ \\
\hline Bradycardic peak (bpm) & $205.3 \pm 2.1$ & $208.7 \pm 7.7$ & 0.8153 \\
\hline Tachycardic peak (bpm) & $346.7 \pm 1.7$ & $467.2 \pm 6$ & $<0.0001$ \\
\hline HR range (bpm) & $150.7 \pm 1.7$ & $258.5 \pm 10.84$ & $<0.0001$ \\
\hline PBG (bpm x mmHg ${ }^{-1}$ ) PHE & $-1.93 \pm 0.196$ & $-2.17 \pm 0.17$ & 0.4561 \\
\hline $\mathrm{SBG}\left(\mathrm{bpm} \times \mathrm{mmHg}^{-1}\right) \mathrm{SNP}$ & $-1.43 \pm 0.06$ & $-2.96 \pm 0.13$ & $<0.0001$ \\
\hline
\end{tabular}
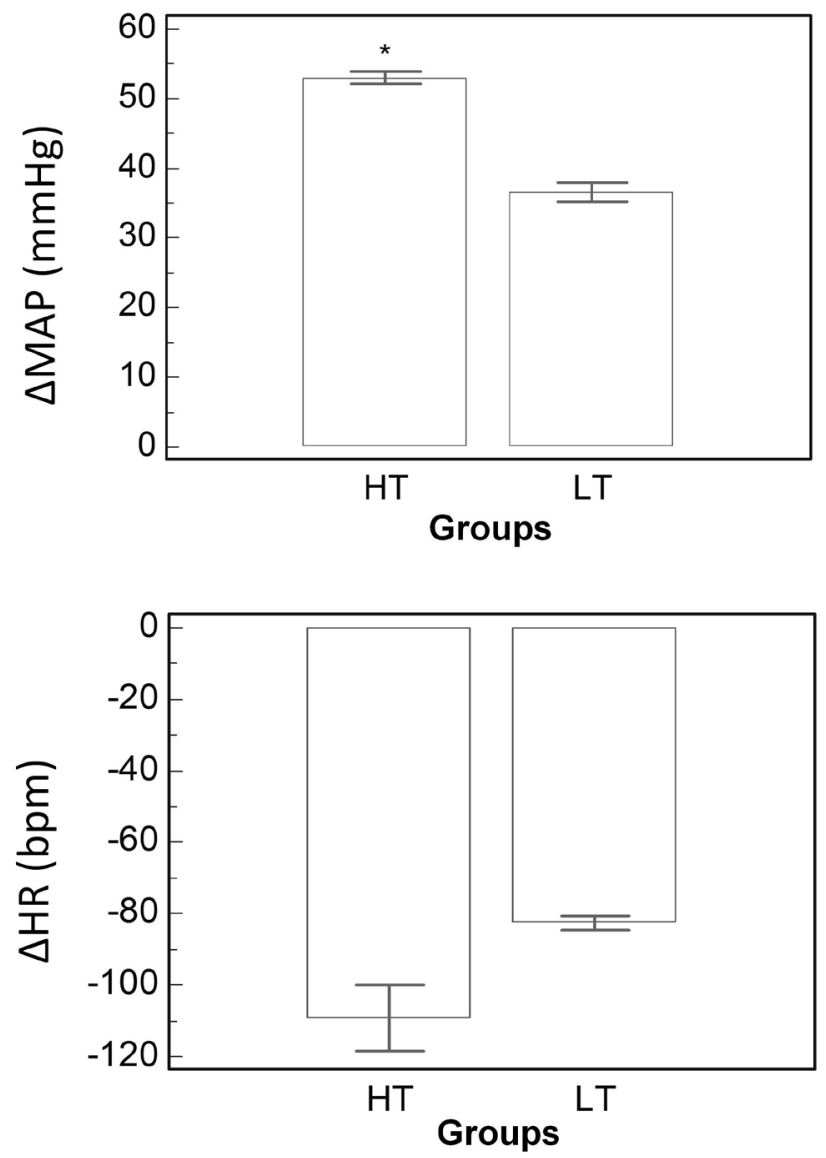

Figure 3 - Increase in mean arterial pressure (MAP, $\mathrm{mmHg}$ ) and decrease in heart rate $(\mathrm{HR}, \mathrm{bpm})$ in response to phenylephrine $(\mathrm{PE}, 8 \mu \mathrm{g} / \mathrm{kg}$ i.v.) in HT $(n=20)$ and LT $(n=6)$ groups. "p<0.001: difference in LT. Means \pm SEM.

The MAP decrease in response to SNP was similar between HT and LT groups ( $\mathrm{p}=0.18$ ) (Figure 4). However, tachycardic reflex responses to a decrease in arterial pressure were significantly reduced in the LT group $(\mathrm{p}<0.001)$.

\section{DISCUSSION}

We compared the baroreceptor reflex among WKY rats
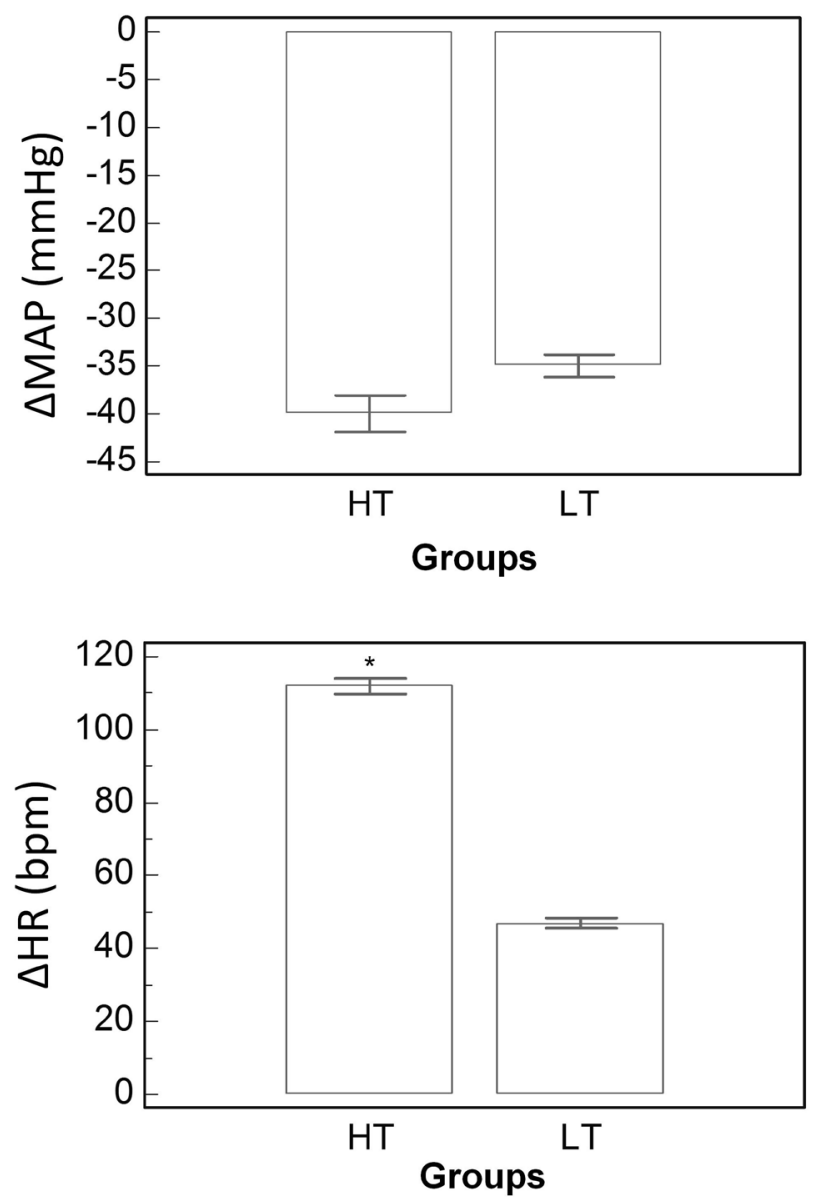

Figure 4 - Decrease in mean arterial pressure (MAP, $\mathrm{mmHg}$ ) and decrease in heart rate $(\mathrm{HR}, \mathrm{bpm})$ in response to sodium nitroprusside (SNP, $50 \mu \mathrm{g} / \mathrm{kg}$ i.v.) in HT $(n=20)$ and LT $(n=6)$ groups. *p<0.001: difference in HT. Means \pm SEM.

from the same laboratory using infusions of the vasopressor $\mathrm{PE}$ and the vasodilator SNP. We found that rats from the same subgroup could be divided into two groups based on baroreflex gain (HB vs. LB, based on the parasympathetic responses and HT vs. LT, and based on the sympathetic responses). Comparison between groups demonstrated that the parasympathetic component of the baroreflex gain and bradycardic reflex response to increased arterial pressure were significantly decreased in approximately $37 \%$ of the animals evaluated. Moreover, the sympathetic component of the baroreflex gain and tachycardic reflex in response to decreased arterial pressure was significantly attenuated in approximately $23 \%$ of the animals studied; this was the same group that was found to have a lower baseline HR. The lack of signs of pain or discomfort suggests that stress or pain after surgery did not influence the outcomes of the experiments.

In our investigation, the baroreflex was estimated by bolus infusion and verified by HR changes in response to arterial pressure elevation or reduction caused by i.v. infusion of PE or SNP, respectively. According to 
our findings, approximately $37 \%$ of 22 WKY rats had a reduced bradycardic peak and bradycardic reflex response to increased arterial pressure as well as attenuated parasympathetic baroreflex gain (LB group). Nonetheless, when we compared basal MAP and HR, tachycardic peak, HR range and sympathetic baroreflex gain, no significant differences were observed. Taken together, these data indicate that a portion of WKY rats exhibit a decrease in reflex bradycardia, the parasympathetic component of baroreflex sensitivity. The mechanisms for reduced function in rats are not completely understood ${ }^{13}$. Some studies have demonstrated that the carotid body is significantly larger in rats with an impaired baroreflex, ${ }^{14-16}$ whereas other studies have shown that the decreased baroreflex function is due to altered levels of norephinephrine, epinephrine and dopamine in the carotid body ${ }^{16-18}$ and medulla oblongata areas that regulate the cardiovascular system. ${ }^{19}$ Furthermore, studies have shown that angiotensin 1 (AT1) receptor densities are involved in impairment of baroreflex function. ${ }^{20,21}$ Similar to our findings were observed for another rat strain (SpragueDawley).${ }^{7}{ }^{10}$ Our findings indicate that baroreflex variability may be normal in rats. Hence, it is not clear whether or not some WKY rats exhibit baroreflex dysfunction or just baroreflex variability.

The observation of great variation in baroreflex sensitivity was surprising because WKY rats are an inbred strain $^{1}$. This intra-strain variation in baroreflex sensitivity in an inbred strain, in which nearly all alleles are homozygotic and the individuals are quite similar, ${ }^{1}$ is supported by a previous study ${ }^{10}$ that showed different growth rates and blood pressures between WKY rates from two different laboratories. However, to the best of our knowledge, no previous investigation has evaluated differences in baroreflex function among WKY rats from the same laboratory.
The baroreceptor reflex was assessed in conscious rather than sedated rats as anesthesia can influence cardiovascular reflex activity ${ }^{22,23}$ and reduce the range of HR, skewing results towards a restricted portion of the WKY rat population. Hence, we believe that our investigation provides accurate information regarding differences in baroreflex function among WKY rats.

Our findings are very relevant, as the baroreceptor reflex is predominantly studied in different models and strains of rats with the goal of preventing the development of hypertension in humans, ${ }^{12,24-26}$ given that reduced baroreflex function is indicative of cardiovascular disease. ${ }^{27-30} \mathrm{We}$ recognize the limitations of our study. A major limitation is that we are unable to provide a full baroreceptor reflex function curve. However, the baroreflex values obtained in our investigation are of physiological relevance, because they fall near the previously reported operating point of this reflex for an unrestrained, conscious rat. ${ }^{31}$

In summary, there was significant variation in baroreflex function among WKY rats from the same laboratory. A majority of the animals presented with enhanced parasympathetic gain of baroreflex (increased bradycardic peak, bradycardic responses to increased arterial pressure and baroreflex gain tested with PE), but a significant minority, approximately $23 \%$, responded with decreased sympathetic activity of the baroreflex (increased tachycardic responses to decreased arterial pressure and increased baroreflex gain tested with SNP).

\section{ACKNOWLEDGEMENTS}

This research was supported by public funding from Fundação de Amparo à Pesquisa do Estado de São Paulo (FAPESP).

\section{REFERENCES}

1. Okamoto K, Aoki K. Development of a strain of spontaneously hypertensive rats. Jpn Circ J. 1963;27:282-93.

2. Kurtz TW, Morris RC Jr. Biological variability in Wistar-Kyoto rats. Implications for research with the spontaneously hypertensive rat. Hypertension. 1987;10:127-31.

3. Guyenet PG. The sympathetic control of blood pressure. Nat Rev Neurosci. 2006;7:335-46.

4. Farah Vde M, De Angelis K, Joaquim LF, Candido GO, Bernardes N, Fazan R Jr, et al. Autonomic modulation of arterial pressure and heart rate variability in hypertensive diabetic rats. Clinics. 2007;62:477-82.

5. Zsotér TT, Nebitko RL, Chow R. The effect of verapamil, diltiazem and nifedipine on baroreceptor reflexes. Clin Invest Med. 1988;11:430-4.
6. La Rovere MT, Bersano C, Gnemmi M, Specchia G, Schwartz PJ Exercise-induced increase in baroreflex sensitivity predicts improved prognosis after myocardial infarction. Circulation. 2002;106:945-9.

7. Mortara A, La Rovere MT, Pinna GD, Prpa A, Maestri R, Febo O, et al. Arterial baroreflex modulation of heart rate in chronic heart failure: clinical and hemodynamic correlates and prognostic implications. Circulation. 1997;96:3450-8

8. Cai GJ, Miao CY, Xie HH, Lu LH, Su DF. Arterial baroreflex dysfunction promotes atherosclerosis in rats. Atherosclerosis. 2005;183:41-7.

9. Shu H, Yi-Ming W, Xu LP, Miao CY, Su DF. Increased susceptibility of ventricular arrhythmia to aconitine in anesthetized rats is attributed to the inhibition of baroreflex. Clin Exp Pharmacol Physiol. 2004;31:249-53. 
10. Liu AJ, Ling G, Wu J, Wang DS, Lin LL, Liu JG, et al. Arterial baroreflex function is an important determinant of acute cerebral ischemia in rats with middle cerebral artery occlusion. Life Sci. 2008;83:388-93.

11. Kurtz TW, Morris RC Jr. Biological variability in Wistar-Kyoto rats. Implications for research with the spontaneously hypertensive rat. Hypertension. 1987;10:127-31.

12. Valenti VE, Ferreira C, Meneghini A, Ferreira M, Murad N, Ferreira Filho $\mathrm{C}$, et al. Evaluation of baroreflex function in young spontaneously hypertensive rats. Arq Bras Cardiol. 2009;92:205-9.

13. Colombari E, Sato MA, Cravo SL, Bergamaschi CT, Campos RR Jr, Lopes OU. Role of the medulla oblongata in hypertension. Hypertension. 2001;38:549-54.

14. Habeck JO, Honig A, Pfeiffer C, Schmidt M. The carotid bodies in spontaneously hypertensive (SHR) and normotensive rats-a study concerning size, location and blood supply. Anat Anz. 1981;150:374-84.

15. Habeck JO, Huckstorf C, Honig A. Influence of age on the carotid bodies of spontaneously hypertensive (SHR) and normotensive rats. I. Arterial blood supply. Exp Pathol. 1984;26:195-203.

16. Pallot DJ, Barer GR. Some observations on the carotid bodies of the New Zealand strain of genetically hypertensive rats. Acta Physiol Pol. 1985;36:65-75.

17. Alho H, Koistinaho J, Kovanen V, Suominen H, Hervonen A. Effect of prolonged physical training on the histochemically demonstrable catecholamines in the sympathetic neurons, the adrenal gland and extra-adrenal catecholamine storing cells of the rat. J Auton Nerv Syst. 1984;10:181-91.

18. Przybylski M, Tripiana J, Gattelet P. Results of an experiment - from unity to division (and back again) Krankenpfl Soins Infirm. 1990;83:5560.

19. Morrison SF, Whitehorn D. Baroreceptor reflex gain is not diminished in spontaneous hypertension. Am J Physiol. 1982;243:R500-5.

20. Veerasingham SJ, Raizada MK. Brain renin-angiotensin system dysfunction in hypertension: recent advances and perspectives. $\mathrm{Br} \mathrm{J}$ Pharmacol. 2003;139:191-202.

21. Gao XY, Zhang F, Han Y, Wang HJ, Zhang Y, Guo R, et al. AT1 receptor in rostral ventrolateral medulla mediating blunted baroreceptor reflex in spontaneously hypertensive rats. Acta Pharmacol Sin. 2004;25:1433-8.
22. Shimokawa A, Kunitake T, Takasaki M, Kannan H. Differential effects of anesthetics on sympathetic nerve activity and arterial baroreceptor reflex in chronically instrumented rats. J Auton Nerv Syst. 1998;72:4654.

23. Fluckiger JP, Sonnay M, Boillat N, Atkinson J. Attenuation of the baroreceptor reflex by general anesthetic agents in the normotensive rat. Eur J Pharmacol. 1985;109:105-9.

24. Souza HC, De Araújo JE, Martins-Pinge MC, Cozza IC, Martins-Dias DP. Nitric oxide synthesis blockade reduced the baroreflex sensitivity in trained rats. Auton Neurosci. 2009;150:38-44.

25. Crestani CC, Deolindo MV, Alves FH, Resstel LB, Correa FM. NonNMDA glutamate receptors in the lateral hypothalamus modulate cardiac baroreflex responses in unanesthetized rats. Clin Exp Pharmacol Physiol. 2009;Apr 27 [Epub ahead of print].

26. Goldman RK, Azar AS, Mulvaney JM, Hinojosa-Laborde C, Haywood JR, Brooks VL. Baroreflex sensitivity varies during the rat estrous cycle: role of gonadal steroids. Am J Physiol Regul Integr Comp Physiol. 2009;296:R1419-26.

27. Souza HC, Ballejo G, Salgado MC, Da Silva VJ, Salgado HC. Cardiac sympathetic overactivity and decreased baroreflex sensitivity in L-NAME hypertensive rats. Am J Physiol Heart Circ Physiol. 2001;280:H844-50.

28. Deley G, Picard G, Taylor JA. Arterial baroreflex control of cardiac vagal outflow in older individuals can be enhanced by aerobic exercise training. Hypertension. 2009;53:826-32.

29. Drolet G, de Champlain J. Hyperresponsiveness of sympathoadrenal system in conscious DOCA-NaCl and SHR rats in response to acute hemorrhagic hypotension. Clin Invest Med. 1992;15:360-70.

30. Kamath MV, Tougas G, Fitzpatrick D, Fallen EL, Watteel R, Shine G, et al. Assessment of the visceral afferent and autonomic pathways in response to esophageal stimulation in control subjects and in patients with diabetes. Clin Invest Med. 1998;21:100-13.

31. Waki H, Katahira K, Polson JW, Kasparov S, Murphy D, Paton JF. Automation of analysis of cardiovascular autonomic function from chronic measurements of arterial pressure in conscious rats. Exp Physiol. 2006;91:201-13. 